Pure and Applied Mathematics Quarterly

Volume 9, Number 3

$547-561,2013$

\title{
Scalar Curvature Pinching for CMC Hypersurfaces in a Sphere
}

\author{
Zhi-Yuan Xu
}

Abstract: We consider an $n$-dimensional closed hypersurface $M$ with
constant mean curvature $H$ in $S^{n+1}, 3 \leq n \leq 8$. Denote by $S$ and
$\beta(n, H)$ the squared norm of the second fundamental form of $M$ and
$S^{1}\left(\frac{1}{\sqrt{1+\mu^{2}}}\right) \times S^{n-1}\left(\frac{\mu}{\sqrt{1+\mu^{2}}}\right)$ respectively, where $\mu=\frac{n|H|+\sqrt{n^{2} H^{2}+4(n-1)}}{2}$. We
prove that there exist two positive constants $\gamma(n)$ and $\epsilon(n)$ such that if
$|H| \leq \gamma(n)$ and $\beta(n, H) \leq S<\beta(n, H)+\epsilon(n)$, then $S \equiv \beta(n, H)$ and $M$ is
one of the following cases: (i) $S^{k}\left(\sqrt{\frac{k}{n}}\right) \times S^{n-k}\left(\sqrt{\frac{n-k}{n}}\right), k=1,2, \ldots, n-1$; (ii)
$S^{1}\left(\frac{1}{\sqrt{1+\mu^{2}}}\right) \times S^{n-1}\left(\frac{\mu}{\sqrt{1+\mu^{2}}}\right)$. This result extends the scalar curvature pinching
theorem for minimal hypersurfaces due to Peng-Terng, Wei-Xu and Zhang. Keywords: hypersurfaces with constant mean curvature, rigidity, scalar curvature, Clifford torus.

\section{INTRODUCTION}

Since the appearance of the famous rigidity theorem for minimal submanifolds due to Simons [15], Lawson [6], and Chern-do Carmo-Kobayashi [4], various scalar curvature pinching problems for submanifolds have been studied by many geometers (see $[3,5,7,8,10,11,12,13,18,20,21,25,29]$, etc.). Let $M$ be an $n$-dimensional closed minimal hypersurface in an $(n+1)$-dimensional unit sphere $S^{n+1}$. Denote by $R$ and $S$ the scalar curvature and the squared length of the second fundamental form of $M$, respectively. From Gauss equation, we have $R=n(n-1)-S$. The Simons-Lawson-Chern-do Carmo-Kobayashi rigidity

Received May 4, 2012.

2000 Mathematics Subject Classification. Primary 53C24; Secondary 53C40. 
theorem says that if $S \leq n$, then $S \equiv 0$ or $S \equiv n$, i.e., $M$ must be the great sphere $S^{n}$ or the Clifford torus $S^{k}\left(\sqrt{\frac{k}{n}}\right) \times S^{n-k}\left(\sqrt{\frac{n-k}{n}}\right), 1 \leq k \leq n-1$. Later, Peng and Terng $[10,11]$ found the second scalar curvature pinching phenomenon for closed minimal hypersurfaces in a unit sphere. In [10], they proved that if the scalar curvature of $M$ is a constant and $n \leq S \leq n+\frac{1}{12 n}$, then $S \equiv n$. When $n=3$, Chang [1] gave the classification of all closed minimal hypersurfaces with constant scalar curvature in $S^{4}$. When $n \geq 4$, Peng-Terng's pinching constant $\frac{1}{12 n}$ was improved to $\frac{n}{3}$ by Yang-Cheng [27, 28], and to $\frac{3 n}{7}$ by Suh-Yang [16], respectively. The classification problem in dimension $n \geq 4$ is still open.

In [11], Peng and Terng obtained an important pinching theorem for $n(\leq 5)$ dimensional minimal hypersurfaces in a unit sphere without assumption that the scalar curvature is a constant.

Theorem 1.1. Let $M^{n}$ be an $n(\leq 5)$-dimensional closed minimal hypersurface in a unit sphere $S^{n+1}$. Then there exists a positive constant $\tau(n)$ depending only on $n$ such that if $n \leq S \leq n+\tau(n)$, then $S \equiv n$, i.e., $M$ is one of the Clifford torus $S^{k}\left(\sqrt{\frac{k}{n}}\right) \times S^{n-k}\left(\sqrt{\frac{n-k}{n}}\right), k=1,2, \ldots, n-1$.

In 1997, Ogiue and Sun [9] claimed that they had generalized Theorem 1.1 to the case for $n \geq 6$. In [17], Wei and Xu pointed out a fatal mistake in the paper by Ogiue and Sun [9]. Moreover, Wei and $\mathrm{Xu}$ [17] solved the second pinching problem for $n=6,7$. Later, Zhang [30] extended the second pinching theorem due to Peng-Terng [11] and Wei-Xu [17] to the case of $n=8$. The second pinching theorem for minimal hypersurfaces due to Wei-Xu-Zhang [17, 30] is stated as follows.

Theorem 1.2. Let $M$ be an n-dimensional closed minimal hypersurface in a unit sphere $S^{n+1}, n=6,7,8$. Then there exists a positive constant $\tau(n)$ depending only on $n$ such that if $n \leq S \leq n+\tau(n)$, then $S \equiv n$, i.e., $M$ is the Clifford torus $S^{k}\left(\sqrt{\frac{k}{n}}\right) \times S^{n-k}\left(\sqrt{\frac{n-k}{n}}\right), 1 \leq k \leq n-1$.

The pinching phenomenon for constant mean curvature hypersurfaces, i.e., CMC hypersurfaces, is much more complicated than the case of minimal hypersurfaces (see [14, 18, 19, 22, 23, 29], etc.). Set

$$
\alpha(n, H)=n+\frac{n^{3} H^{2}}{2(n-1)}-\frac{n(n-2)}{2(n-1)} \sqrt{n^{2} H^{4}+4(n-1) H^{2}},
$$




$$
\begin{gathered}
\beta(n, H)=n+\frac{n^{3} H^{2}}{2(n-1)}+\frac{n(n-2)}{2(n-1)} \sqrt{n^{2} H^{4}+4(n-1) H^{2}}, \\
\lambda=\frac{n|H|+\sqrt{n^{2} H^{2}+4(n-1)}}{2(n-1)}, \quad \mu=\frac{n|H|+\sqrt{n^{2} H^{2}+4(n-1)}}{2} .
\end{gathered}
$$

In [18], Xu proved the following pinching theorem for submanifolds with parallel mean curvature in a sphere.

Theorem 1.3. Let $M$ be an $n$-dimensional compact submanifold with parallel mean curvature vector $(H \neq 0)$ in an $(n+p)$-dimensional unit sphere $S^{n+p}$. If $S \leq \alpha(n, H)$, then either $M$ is pseudo-umbilical, or $S \equiv \alpha(n, H)$ and $M$ is the isoparametric hypersurface $S^{n-1}\left(\frac{1}{\sqrt{1+\lambda^{2}}}\right) \times S^{1}\left(\frac{\lambda}{\sqrt{1+\lambda^{2}}}\right)$ in a great sphere $S^{n+1}$. In particular, if $M$ is a compact hypersurface with constant mean curvature $H(\neq 0)$ in $S^{n+1}$, then $M$ is either a totally umbilical sphere $S^{n}\left(\frac{1}{\sqrt{1+H^{2}}}\right)$, or a Clifford hypersurface $S^{n-1}\left(\frac{1}{\sqrt{1+\lambda^{2}}}\right) \times S^{1}\left(\frac{\lambda}{\sqrt{1+\lambda^{2}}}\right)$.

In [23], Xu and Tian generalized Suh-Yang's pinching theorem [16] to the case where $M$ is a compact hypersurface with constant scalar curvature and small constant mean curvature in $S^{n+1}$. The following second pinching theorem for hypersurfaces with small constant mean curvature was proved by Cheng-He-Li [2] and Xu-Zhao [24], respectively.

Theorem 1.4. Let $M$ be an $n$-dimensional closed hypersurface in a unit sphere $S^{n+1}$ with constant mean curvature $H(\neq 0), 3 \leq n \leq 7$. There exist two positive constants $\gamma(n)$ and $\epsilon(n)$ such that if $|H| \leq \gamma(n)$, and $\beta(n, H) \leq S<\beta(n, H)+$ $\epsilon(n)$, then $S \equiv \beta(n, H)$ and $M=S^{1}\left(\frac{1}{\sqrt{1+\mu^{2}}}\right) \times S^{n-1}\left(\frac{\mu}{\sqrt{1+\mu^{2}}}\right)$.

In this paper, we obtain the following theorem which extends Theorem 1.2 and Theorem 1.4.

Theorem 1.5 (Main Theorem). Let $M$ be an n-dimensional closed hypersurface in a unit sphere $S^{n+1}$ with constant mean curvature $H, 3 \leq n \leq 8$. There exist two positive constants $\gamma(n)$ and $\epsilon(n)$ depending only on $n$ such that if $|H| \leq \gamma(n)$, and $\beta(n, H) \leq S<\beta(n, H)+\epsilon(n)$, then $S \equiv \beta(n, H)$ and $M$ is one of the following cases: (i) $S^{k}\left(\sqrt{\frac{k}{n}}\right) \times S^{n-k}\left(\sqrt{\frac{n-k}{n}}\right), 1 \leq k \leq n-1$; (ii) $S^{1}\left(\frac{1}{\sqrt{1+\mu^{2}}}\right) \times$ $S^{n-1}\left(\frac{\mu}{\sqrt{1+\mu^{2}}}\right)$. 


\section{Preliminaries}

Let $M^{n}$ be an $n$-dimensional closed hypersurface with constant mean curvature in a unit sphere $S^{n+1}$. We shall make use of the following convention on the range of indices.

$$
\begin{gathered}
1 \leq A, B, C, \ldots, \leq n+1, \\
1 \leq i, j, k, \ldots, \leq n .
\end{gathered}
$$

Choose an orthonormal frame field $\left\{e_{A}\right\}$ in a neighborhood of $p \in M$ such that the $e_{i}$ 's are tangent to $M$ at $p$. Let $\left\{\omega_{A}\right\}$ be the dual frame fields of $\left\{e_{A}\right\}$ and $\left\{\omega_{A B}\right\}$ be the connection 1-forms of $S^{n+1}$. Restricting to $M$, we have

$$
\omega_{n+1 j}=\sum_{i} h_{j i} \omega_{i}, \quad h_{j i}=h_{i j}
$$

Let $R$ and $h$ be the scalar curvature and the second fundamental form of $M$, respectively. Denote by $S$ the squared length of $h$ and $H$ the mean curvature of $M$, and assume $H \geq 0$. Then we have

$$
\begin{gathered}
h=\sum_{i, j} h_{i j} \omega_{i} \otimes \omega_{j}, \quad S=\sum_{i, j} h_{i j}^{2}, \\
H=\frac{1}{n} \sum_{i} h_{i i}, \\
R=n(n-1)+n^{2} H^{2}-S .
\end{gathered}
$$

Denote by $h_{i j k}, h_{i j k l}$ and $h_{i j k l m}$ the first, second and third covariant derivatives of the second fundamental tensor $h_{i j}$. Then

$$
\begin{gathered}
\nabla h=\sum_{i, j, k} h_{i j k} \omega_{i} \otimes \omega_{j} \otimes \omega_{k}, h_{i j k}=h_{i k j}, \\
h_{i j k l}=h_{i j l k}+\sum_{m} h_{m j} R_{m i k l}+\sum_{m} h_{i m} R_{m j k l}, \\
h_{i j k l m}=h_{i j k m l}+\sum_{r} h_{r j k} R_{r i l m}+\sum_{r} h_{i r k} R_{r j l m}+\sum_{r} h_{i j r} R_{r k l m} .
\end{gathered}
$$


For an arbitrary fixed point $x \in M$, we take an orthonormal frame $\left\{e_{i}\right\}$ such that $h_{i j}=\lambda_{i} \delta_{i j}$ for all $i, j$. Then $\sum_{i} \lambda_{i}=n H$, and $\sum_{i} \lambda_{i}^{2}=S$. By a similar computation as in [11], we obtain

$$
\frac{1}{2} \Delta S=S(n-S)-n^{2} H^{2}+n H f_{3}+|\nabla h|^{2},
$$

and

$$
\begin{aligned}
\frac{1}{2} \Delta|\nabla h|^{2}= & (2 n+3-S)|\nabla h|^{2}-\frac{3}{2}|\nabla S|^{2}+\left|\nabla^{2} h\right|^{2} \\
& +\sum_{i, j, k, l, m}\left(6 h_{i j k} h_{i l m} h_{j l} h_{k m}-3 h_{i j k} h_{i j l} h_{k m} h_{m l}\right) \\
& +3 n H \sum_{i, j, k, l} h_{i j k} h_{j l k} h_{l i} \\
= & (2 n+3-S)|\nabla h|^{2}-\frac{3}{2}|\nabla S|^{2}+\left|\nabla^{2} h\right|^{2} \\
& +3(2 B-A)+3 n H C,
\end{aligned}
$$

where

$$
f_{k}=\sum_{i} \lambda_{i}^{k}, A=\sum_{i, j, k} h_{i j k}^{2} \lambda_{i}^{2}, B=\sum_{i, j, k} h_{i j k}^{2} \lambda_{i} \lambda_{j}, C=\sum_{i, j, k} h_{i j k}^{2} \lambda_{i} .
$$

From [10], we have

$$
h_{i j i j}=h_{j i j i}+t_{i j},
$$

where $t_{i j}=\left(\lambda_{i}-\lambda_{j}\right)\left(1+\lambda_{i} \lambda_{j}\right)$. This implies

$$
\left|\nabla^{2} h\right|^{2} \geq \frac{3}{4} \sum_{i \neq j} t_{i j}^{2}=\frac{3}{4} \sum_{i, j} t_{i j}^{2} .
$$

Hence

$$
\left|\nabla^{2} h\right|^{2} \geq \frac{3}{2}\left[S f_{4}-f_{3}^{2}-S^{2}-S(S-n)-n^{2} H^{2}+2 n H f_{3}\right]
$$


Now we give a similar computation as in [11].

$$
\begin{aligned}
\frac{1}{3} \sum_{i, j} h_{i j}\left(f_{3}\right)_{i j} & =\frac{1}{3} \sum_{k} \lambda_{k}\left(f_{3}\right)_{k k} \\
& =\sum_{k} \lambda_{k}\left(\sum_{i} h_{i i k k} \lambda_{i}^{2}+2 \sum_{i, j} h_{i j k}^{2} \lambda_{i}\right) \\
& =\sum_{i, k} h_{i i k k} \lambda_{k} \lambda_{i}^{2}+2 \sum_{i, j, k} h_{i j k}^{2} \lambda_{i} \lambda_{k} \\
& =\sum_{i, k}\left[h_{k k i i}+\left(\lambda_{i}-\lambda_{k}\right)\left(1+\lambda_{i} \lambda_{k}\right)\right] \lambda_{k} \lambda_{i}^{2}+2 B \\
& =\sum_{i}\left(\frac{S_{i i}}{2}-\sum_{j, k} h_{i j k}^{2}\right) \lambda_{i}^{2}+\sum_{i, k} \lambda_{i}^{2} \lambda_{k}\left(\lambda_{i}-\lambda_{k}\right)\left(1+\lambda_{i} \lambda_{k}\right)+2 B \\
& =\sum_{i, j, k} \frac{h_{i k} h_{k j}}{2} S_{i j}+n H f_{3}-S^{2}-f_{3}^{2}+S f_{4}-(A-2 B) .
\end{aligned}
$$

Since $\int_{M} \sum_{i, j} h_{i j}\left(f_{3}\right)_{i j} d M=0$, we drive following integral equation of $A-2 B$.

$$
\begin{aligned}
\int_{M}(A-2 B) d M= & \int_{M}\left[n H f_{3}-S^{2}-f_{3}^{2}+S f_{4}+\sum_{i, j, k} \frac{h_{i k} h_{k j}}{2} S_{i j}\right] d M \\
= & \int_{M}\left[n H f_{3}-S^{2}-f_{3}^{2}+S f_{4}-\sum_{i, j, k}\left(h_{i k} h_{k j}\right)_{j} \frac{S_{i}}{2}\right] d M \\
= & \int_{M}\left[n H f_{3}-S^{2}-f_{3}^{2}+S f_{4}-\sum_{i, j, k} h_{i k j} h_{k j} \frac{S_{i}}{2}\right. \\
& \left.-\sum_{i, j, k} h_{i k} h_{k j j} \frac{S_{i}}{2}\right] d M \\
= & \int_{M}\left[n H f_{3}-S^{2}-f_{3}^{2}+S f_{4}-\sum_{i, j, k} h_{i k j} h_{k j} \frac{S_{i}}{2}\right] d M \\
= & \int_{M}\left[n H f_{3}-S^{2}-f_{3}^{2}+S f_{4}-\frac{|\nabla S|^{2}}{4}\right] d M .
\end{aligned}
$$


From (2.8) (2.9) (2.12) (2.14), we have

$$
\begin{aligned}
\int_{M}\left|\nabla^{2} h\right|^{2} d M & =\int_{M}\left[(S-2 n-3)|\nabla h|^{2}+\frac{3}{2}|\nabla S|^{2}+3(A-2 B)-3 n H C\right] d M \\
& \geq \int_{M} \frac{3}{2}\left[S f_{4}-f_{3}^{2}-S^{2}-S(S-n)-n^{2} H^{2}+2 n H f_{3}\right] d M \\
& =\int_{M}\left[\frac{3}{2}(A-2 B)-\frac{3}{2}|\nabla h|^{2}+\frac{3}{8}|\nabla S|^{2}\right] d M .
\end{aligned}
$$

Hence

$$
\int_{M}\left[\left(S-2 n-\frac{3}{2}\right)|\nabla h|^{2}+\frac{3}{2}(A-2 B)+\frac{9}{8}|\nabla S|^{2}-3 n H C\right] d M \geq 0 .
$$

\section{Proof of MAIN THEOREM}

To simplify the computation, we use the tracefree second fundamental form $\phi=\sum_{i, j} \phi_{i j} \omega_{i} \otimes \omega_{j}$, where $\phi_{i j}=h_{i j}-H \delta_{i j}$. If $h_{i j}=\lambda_{i} \delta_{i j}$, then $\phi_{i j}=\mu_{i} \delta_{i j}$, where $\mu_{i}=\lambda_{i}-H$. Putting $\Phi=|\phi|^{2}$ and $\bar{f}_{k}=\sum_{i} \mu_{i}^{k}$, we get $\Phi=S-n H^{2}$ and $f_{3}=\bar{f}_{3}+3 H \Phi+n H^{3}$. Then, we rewrite (2.16) as follows.

$$
\int_{M}\left[\left(\Phi+n H^{2}-2 n-\frac{3}{2}\right)|\nabla \phi|^{2}+\frac{3}{2}(A-2 B)+\frac{9}{8}|\nabla \Phi|^{2}-3 n H C\right] d M \geq 0 .
$$

On the other hand, since $\int_{M} \frac{1}{4} \Delta \Phi^{2} d M=0$, from (2.8), we have

$$
-\frac{1}{2} \int_{M}|\nabla \Phi|^{2} d M=\int_{M}\left[\Phi|\nabla \phi|^{2}-\Phi^{3}+n \Phi^{2}+n H^{2} \Phi^{2}+n H \overline{f_{3}} \Phi\right] d M .
$$

From (3.1) and (3.2), we have

$$
\begin{aligned}
0 \leq & \int_{M}\left[\left(\Phi+n H^{2}-2 n-\frac{3}{2}\right)|\nabla \phi|^{2}+\frac{3}{2}(A-2 B)\right. \\
& \left.-\frac{9}{4}\left(\Phi|\nabla \phi|^{2}-\Phi^{3}+n \Phi^{2}+n H^{2} \Phi^{2}+n H \Phi \sum_{i} \mu_{i}^{3}\right)-3 n H C\right] d M \\
= & \int_{M}\left[\left(-\frac{5}{4} \Phi+n H^{2}-2 n-\frac{3}{2}\right)|\nabla \phi|^{2}+\frac{3}{2}(A-2 B)\right. \\
& \left.+\frac{9}{4} \Phi F(\Phi)-3 n H C\right] d M,
\end{aligned}
$$

where

$$
F(\Phi)=\Phi^{2}-n \Phi-n H^{2} \Phi-n H \sum_{i} \mu_{i}^{3}
$$


Lemma $3.1([18])$. Let $a_{1}, a_{2}, \ldots, a_{n}$ be real numbers satisfying $\sum_{i} a_{i}=0$ and $\sum_{i} a_{i}^{2}=a$. Then

$$
\left|\sum_{i} a_{i}^{3}\right| \leq \frac{n-2}{\sqrt{n(n-1)}} a^{\frac{3}{2}}
$$

and the equality holds if and only if at least $n-1$ numbers of $a_{i}$ 's are same with each other.

From Lemma 3.1, we get

$$
\begin{aligned}
F(\Phi) & \geq \Phi^{2}-n \Phi-n H^{2} \Phi-n H \frac{(n-2) \Phi^{\frac{3}{2}}}{\sqrt{n(n-1)}} \\
& =\Phi\left[\Phi-n H \frac{(n-2) \Phi^{\frac{1}{2}}}{\sqrt{n(n-1)}}-n\left(1+H^{2}\right)\right] \\
& \geq 0,
\end{aligned}
$$

where

$$
\Phi \geq \beta_{0}(n, H):=n+\frac{n^{3}}{2(n-1)} H^{2}+\frac{n(n-2)}{2(n-1)} \sqrt{n^{2} H^{4}+4(n-1) H^{2}}-n H^{2} .
$$

Moreover, if $F(\Phi)=0$, then $\Phi=\beta_{0}(n, H)$ and at least $n-1$ numbers of $\mu_{i}$ 's are same with each other.

We consider the case for $\beta_{0}(n, H) \leq \Phi<\beta_{0}(n, H)+\epsilon(n)$, where $\epsilon(n)$ is to be determined. From (2.8), we have

$$
\int_{M} F(\Phi) d M=\int_{M}|\nabla \phi|^{2} d M
$$

Then

$$
\int_{M} \Phi F(\Phi) d M \leq \int_{M}\left(\beta_{0}(n, H)+\epsilon(n)\right)|\nabla \phi|^{2} d M .
$$

Since $|C| \leq \sqrt{S}|\nabla h|^{2} \leq S|\nabla h|^{2}$, from (3.3) and (3.6), we have

$$
\begin{aligned}
0 \leq & \int_{M}\left[\left(-\frac{5}{4} \Phi+n H^{2}-2 n-\frac{3}{2}+3 n H \Phi+3 n^{2} H^{3}\right)|\nabla \phi|^{2}+\frac{3}{2}(A-2 B)\right. \\
& \left.+\frac{9}{4}\left(\beta_{0}(n, H)+\epsilon(n)\right)|\nabla \phi|^{2}\right] d M .
\end{aligned}
$$

Lemma 3.2. Let $M$ be an $n$-dimensional closed hypersurface in a unit sphere $S^{n+1}$ with constant mean curvature, $n \geq 4$. Suppose that

$$
3(A-2 B) \leq t(n) S|\nabla h|^{2},
$$


where $t(n)$ is a number depending only on $n$, satisfying $0 \leq t(n)<2+\frac{3}{n}$. Then there exist positive constants $\gamma(n)$ and $\epsilon(n)$ such that if $|H| \leq \gamma(n)$, and $\beta(n, H) \leq$ $S<\beta(n, H)+\epsilon(n)$, then $S \equiv \beta(n, H)$. Here

$$
\beta(n, H)=n+\frac{n^{3}}{2(n-1)} H^{2}+\frac{n(n-2)}{2(n-1)} \sqrt{n^{2} H^{4}+4(n-1) H^{2}} .
$$

Proof. Putting $D(n, H)=\beta_{0}(n, H)+n H^{2}-n$, from (3.7) and the condition of the lemma, we have

$$
\begin{aligned}
0 \leq & \int_{M}\left[\left(-\frac{5}{4} \Phi+n H^{2}-2 n-\frac{3}{2}+3 n H \Phi+3 n^{2} H^{3}\right)|\nabla \phi|^{2}+\frac{t(n)}{2} S|\nabla \phi|^{2}\right. \\
& \left.+\frac{9}{4}\left(\beta_{0}(n, H)+\epsilon(n)\right)|\nabla \phi|^{2}\right] d M \\
= & \int_{M}\left[\left(-\frac{5}{4}+3 n H+\frac{t(n)}{2}\right)\left(\Phi-\beta_{0}(n, H)\right)\right. \\
& +\left(-\frac{5}{4}+3 n H+\frac{t(n)}{2}+\frac{9}{4}\right) \beta_{0}(n, H) \\
& \left.+n H^{2}-2 n-\frac{3}{2}+3 n^{2} H^{3}+\frac{t(n)}{2} n H^{2}+\frac{9}{4} \epsilon(n)\right]|\nabla \phi|^{2} d M \\
= & \int_{M}\left[\left(-\frac{5}{4}+3 n H+\frac{t(n)}{2}\right)\left(\Phi-\beta_{0}(n, H)\right)\right. \\
& +\left(1+3 n H+\frac{t(n)}{2}\right)\left(n+D(n, H)-n H^{2}\right) \\
& \left.+n H^{2}-2 n-\frac{3}{2}+3 n^{2} H^{3}+\frac{t(n)}{2} n H^{2}+\frac{9}{4} \epsilon(n)\right]|\nabla \phi|^{2} d M \\
= & \int_{M}\left[\left(-\frac{5}{4}+3 n H+\frac{t(n)}{2}\right)\left(\Phi-\beta_{0}(n, H)\right)+\left(1+3 n H+\frac{t(n)}{2}\right) D(n, H)\right. \\
3.8) & \left.+3 n^{2} H+\left(1+\frac{t(n)}{2}\right) n-2 n-\frac{3}{2}+\frac{9}{4} \epsilon(n)\right]|\nabla \phi|^{2} d M .
\end{aligned}
$$

Since $t(n)<2+\frac{3}{n}$, we have

$$
\begin{aligned}
\left(1+\frac{t(n)}{2}\right) n-2 n-\frac{3}{2} & <\left(1+\frac{2+\frac{3}{n}}{2}\right) n-2 n-\frac{3}{2} \\
& =2 n+\frac{3}{2}-2 n-\frac{3}{2} \\
& =0 .
\end{aligned}
$$

Letting $E(n, H)=\left(1+3 n H+\frac{t(n)}{2}\right) D(n, H)+3 n^{2} H$, we see that $E(n, t)$ is strictly increasing for $t \geq 0$ and $E(n, 0)=0$. Hence there exists a positive constant $\gamma^{\prime}(n)$, 
such that if $H \leq \gamma^{\prime}(n)$, then

$$
\left(1+3 n H+\frac{t(n)}{2}\right) D(n, H)+3 n^{2} H \leq \frac{1}{2}\left[2 n+\frac{3}{2}-\left(1+\frac{t(n)}{2}\right) n\right] .
$$

Set $\gamma^{\prime \prime}(n)=\frac{1-\frac{3}{n}}{12 n}$ and

$$
\epsilon(n, H)=-\frac{4}{9}\left[\left(1+3 n H+\frac{t(n)}{2}\right) D(n, H)+3 n^{2} H+\left(1+\frac{t(n)}{2}\right) n-2 n-\frac{3}{2}\right] .
$$

When $n \geq 4$ and $H \leq \gamma^{\prime \prime}(n)$, we have

$$
\begin{aligned}
-\frac{5}{4}+3 n H+\frac{t(n)}{2} & <-\frac{5}{4}+3 n H+\frac{2+\frac{3}{n}}{2} \\
& =3 n H+\frac{\frac{3}{n}-1}{4} \\
& \leq 0 .
\end{aligned}
$$

Take $\gamma(n)=\min \left\{\gamma^{\prime}(n), \gamma^{\prime \prime}(n)\right\}$ and $\epsilon(n)=\epsilon(n, \gamma(n))$. When $H \leq \gamma(n)$ and $\beta(n, H) \leq S<\beta(n, H)+\epsilon(n)$, combining (3.8) and (3.10), we have

$$
\int_{M}\left(-\frac{5}{4}+3 n H+\frac{t(n)}{2}\right)\left(\Phi-\beta_{0}(n, H)\right)|\nabla \phi|^{2} d M=0 .
$$

Putting $X=\left\{x \in M \mid \Phi(x)>\beta_{0}(n, H)\right\}$ and $Y=\left\{x \in M \mid \Phi(x)=\beta_{0}(n, H)\right\}$, we have $M=X \cup Y$. We assert that $X=\emptyset$. Otherwise, from (3.11), we have $\nabla \phi(x)=\nabla h(x)=0$ for $x \in X$. Since $\Phi$ is a continuous function on $M$, $X=\left\{x \in M \mid \Phi(x)>\beta_{0}(n, H)\right\}$ is an open subset. Hence $\Phi(x)$ identically equals to a constant $\tau_{0}$ on $X$. This shows that $X$ is a closed subset. So, $X=M$. This together with (3.11) implies that $\nabla \phi(x)=\nabla h(x)=0$ for all $x \in M$. It follows from (3.4) and (3.5) that $\Phi(x) \equiv \beta_{0}(n, H)$. This is a contradiction. Therefore, $M=Y$, i.e., $\Phi \equiv \beta_{0}(n, H)$. This implies $S \equiv \beta(n, H)$.

By using a similar method as in the proof of Lemma 3.2 in [30], we get the following lemma.

Lemma 3.3. Let $M$ be an n-dimensional closed hypersurface with constant mean curvature in a unit sphere $S^{n+1}$. If $\lambda_{1}^{2}-4 \lambda_{1} \lambda_{2} \geq t S$ for some $t \geq 2$, then $\left(\lambda_{1}^{2}-4 \lambda_{1} \lambda_{2}\right)-\left(\lambda_{1}^{2}-4 \lambda_{1} \lambda_{i}\right) \geq r S$, where $r=\frac{16 t-8-12 \sqrt{-2 t^{2}+2 t+8}}{17}$ and $i \neq 1,2$.

The following lemma will be used in proof of Lemma 3.5. 
Lemma $3.4([30])$. Let $f_{n}(t)=17[t-2-\delta(n)][3(n-2) t+(n+2) \delta(n)+10-4 n]$ and $g_{n}(t)=[8+16 \delta(n)]\left(4 t-2-3 \sqrt{-2 t^{2}+2 t+8}\right)$. Then

$$
h_{n}(t)=f_{n}(t)-g_{n}(t) \leq 0,
$$

where $t \geq 2, n=8, \delta(8)=0.34$.

Putting $b_{i}=h_{i i 1}, b=\sum_{i \neq 1} b_{i}^{2}+\frac{1}{3} b_{1}^{2}, f=\sum_{i \neq 1}\left(\lambda_{1}^{2}-4 \lambda_{1} \lambda_{i}\right) b_{i}^{2}-\lambda_{1}^{2} b_{1}^{2}$, we have

Lemma 3.5. Let $M$ be an 8-dimensional closed hypersurface with constant mean curvature in a unit sphere $S^{9}$. Then

$$
f \leq(2+\delta(n)) S b
$$

where $n=8, \delta(8)=0.34$.

Proof. If $\lambda_{1}^{2}-4 \lambda_{1} \lambda_{i} \leq(2+\delta(n)) S$ for $2 \leq i \leq n$, then we have proven Lemma 3.5. Otherwise, without loss of generality, we assume that $\lambda_{1}^{2}-4 \lambda_{1} \lambda_{2}=t S$ for some $t \geq 2$, and $b_{1}=x b_{2}$. Since $H$ is a constant, we have $\left(b_{1}+b_{2}\right)^{2}=\left(\sum_{i \neq 1,2} b_{i}\right)^{2}$. Hence

$$
\begin{gathered}
\sum_{i \neq 1,2} b_{i}^{2} \geq \frac{(1+x)^{2}}{n-2} b_{2}^{2}, \\
\lambda_{1}^{2} \geq(t-2) S .
\end{gathered}
$$

From (3.12), (3.13) and Lemma 3.3, we have

$$
\begin{aligned}
f-(2+\delta(n)) S b \leq & (t-2-\delta(n)) S b_{2}^{2}+(t-r-2-\delta(n)) S \sum_{i \neq 1,2} b_{i}^{2} \\
& -\left(t-2+\frac{2+\delta(n)}{3}\right) S b_{1}^{2} \\
\leq & (t-2-\delta(n)) S b_{2}^{2}+\frac{t-r-2-\delta(n)}{n-2}(1+x)^{2} S b_{2}^{2} \\
& -\left(t-2+\frac{2+\delta(n)}{3}\right) x^{2} S b_{2}^{2},
\end{aligned}
$$

where $r=\frac{16 t-8-12 \sqrt{-2 t^{2}+2 t+8}}{17}$. Let $F(n, t, x)=t-2-\delta(n)+\frac{t-r-2-\delta(n)}{n-2}(1+x)^{2}-$ $\left(t-2+\frac{2+\delta(n)}{3}\right) x^{2}$, then $(3.14)$ becomes

$$
f-(2+\delta(n)) S b \leq F(n, t, x) S b_{2}^{2}
$$


For fixed $t$, let $x_{0}$ be the maximum point of $F(n, t, x)$. Noting that $\left.\frac{\partial F(n, t, x)}{\partial x}\right|_{x=x_{0}}=$ 0 , we have

$$
F(n, t, x) \leq F\left(n, t, x_{0}\right)=\frac{h_{n}(t)}{51 G(n, t)},
$$

where $G(n, t)=r+2+\delta(n)-t+(n-2)\left(t-2+\frac{2+\delta(n)}{3}\right)$, and $h_{n}(t)$ is defined in Lemma 3.4. Therefore,

$$
f \leq(2+\delta(n)) S b
$$

Lemma 3.6. Let $M$ be an 8-dimensional closed hypersurface with constant mean curvature in a unit sphere $S^{9}$. Then

$$
3(A-2 B) \leq 2.34 S|\nabla h|^{2},
$$

where $A, B$ are defined in (2.9).

Proof. Using Lemma 3.5, for any $j$, we have

$$
\begin{aligned}
f_{j} & =\sum_{i \neq j}\left(\lambda_{j}^{2}-4 \lambda_{j} \lambda_{i}\right) h_{i i j}^{2}-\lambda_{j}^{2} h_{j j j}^{2} \\
& \leq(2+\delta(n)) S\left(\sum_{i \neq j} h_{i i j}^{2}+\frac{1}{3} h_{j j j}^{2}\right) .
\end{aligned}
$$

Therefore

$$
\begin{aligned}
3(A-2 B)= & \sum_{i, j, k \text { distinct }}\left[2\left(\lambda_{i}^{2}+\lambda_{j}^{2}+\lambda_{k}^{2}\right)-\left(\lambda_{i}+\lambda_{j}+\lambda_{k}\right)^{2}\right] h_{i j k}^{2} \\
& -3 \sum_{i} \lambda_{i}^{2} h_{i i i}^{2}+3 \sum_{j} \sum_{i \neq j}\left(\lambda_{j}^{2}-4 \lambda_{i} \lambda_{j}\right) h_{i i j}^{2} \\
\leq & 2 S \sum_{i, j, k \text { distinct }} h_{i j k}^{2}+3 \sum_{j} \sum_{i \neq j}\left[\left(\lambda_{j}^{2}-4 \lambda_{i} \lambda_{j}\right) h_{i i j}^{2}-\lambda_{j}^{2} h_{j j j}^{2}\right] \\
\leq & (2+\delta(n)) S\left(\sum_{i, j, k \text { distinct }} h_{i j k}^{2}+3 \sum_{j} \sum_{i \neq j} h_{i i j}^{2}+\sum_{j} h_{j j j}^{2}\right) \\
= & (2+\delta(n)) S \sum_{i, j, k} h_{i j k}^{2} .
\end{aligned}
$$

Since $\delta(8)=0.34$, we have

$$
3(A-2 B) \leq 2.34 S|\nabla h|^{2} .
$$


Now we are in a position to give the proof of Main Theorem.

Proof. i) When $3 \leq n \leq 7$, Main Theorem follows from Theorem 1.1, Theorem 1.2 and Theorem 1.4.

ii) When $n=8$, from Lemma 3.2, Lemma 3.6 and the condition of Main Theorem, we have

$$
S \equiv \beta(n, H)
$$

Hence

$$
F(\Phi)=0 .
$$

From Lemma 3.1, we know that at least $n-1$ numbers of the $\lambda_{i}$ 's are same with each other. From the condition of Main Theorem, we have

$$
\begin{gathered}
\lambda_{1}=\cdots=\lambda_{n-1}=H-\sqrt{\frac{\beta(n, H)-n H^{2}}{n(n-1)}}, \\
\lambda_{n}=H+\sqrt{\frac{(n-1)\left(\beta(n, H)-n H^{2}\right)}{n}} .
\end{gathered}
$$

Therefore $M$ is the Clifford hypersurface

$$
S^{1}\left(\frac{1}{\sqrt{1+\mu^{2}}}\right) \times S^{n-1}\left(\frac{\mu}{\sqrt{1+\mu^{2}}}\right)
$$

in $S^{n+1}$, where $\mu=\frac{n H+\sqrt{n^{2} H^{2}+4(n-1)}}{2}$. This completes the proof of Main Theorem.

\section{ACKnowledgements}

This paper forms part of the author's Bachelor thesis [26] in S.-T. Yau Mathematics Elite Class at Zhejiang University. This thesis was awarded the New World Mathematics Awards in 2010. The author would like to express his deep gratitude to Professor Kefeng Liu, Professor Hong-Wei Xu and Professor ShingTung Yau for their constant encouragements and important helps.

\section{REFERENCES}

1. S.-P. Chang: On minimal hypersurfaces with constant scalar curvatures in $S^{4}$, J. Differential Geom. 37(1993), 523-534.

2. Q.-M. Cheng, Y.-J. He and H.-Z. Li: Scalar curvature of hypersurfaces with constant mean curvature in a sphere, Glasgow Math. J. 51(2009), 413-423. 
3. S.-Y. Cheng and S.-T. Yau: Hypersurfaces with constant scalar curvature, Math. Ann. 225(1977), 195-204.

4. S.-S. Chern, M. do Carmo, and S. Kobayashi: Minimal submanifolds of a sphere with second fundamental form of constant length, in Functional Analysis and Related Fields, SpringerVerlag, New York, 1970.

5. Q. Ding: On spectral characterizations of minimal hypersurfaces in a sphere, Kodai Math. J. 17(1994), 320-328.

6. B. Lawson: Local rigidity theorems for minimal hypersurfaces, Ann. of Math. 89(1969), 187-197.

7. A.-M. Li and J.-M. Li: An intrinsic rigidity theorem for minimal submanifolds in a sphere, Arch. Math. 58(1992), 582-594.

8. H.-Z. Li: Hypersurfaces with constant scalar curvature in space forms, Math. Ann. 305(1996), 665-672.

9. K. Ogiue and H.-F. Sun: Minimal hypersurfaces of unit sphere, Tohoku Math. J. 49(1997), 423-429.

10. C.-K. Peng and C.-L. Terng: Minimal hypersurfaces of sphere with constant scalar curvature, Ann. of Math. Study. 103(1983), 177-198.

11. C.-K. Peng and C.-L. Terng: The scalar curvature of minimal hypersurfaces in spheres, Math. Ann. 266(1983), 105-113.

12. Y.-B. Shen: On intrinsic rigidity for minimal submanifolds in a sphere, Sci. Sinica Ser. A 32(1989), 769-781.

13. K. Shiohama and H.-W. Xu: The topological sphere theorem for complete submanifolds, Compositio Math. 107(1997), 221-232.

14. K. Shiohama and H.-W. Xu: A general rigidity theorem for complete submanifolds, Nagoya Math. J. 150(1998), 105-134.

15. J. Simons: Minimal varieties in Riemannian manifolds, Ann. of Math. 88(1968), 62-105.

16. Y.-J. Suh and H.-Y. Yang: The scalar curvature of minimal hypersurfaces in a unit sphere, Comm. Contemporary Math. 9(2007), 183-200.

17. S.-M. Wei and H.-W. Xu: Scalar curvature of minimal hypersurfaces in a sphere, Math. Res. Lett. 14(2007), 423-432.

18. H.-W. Xu: A rigidity theorem for submanifolds with parallel mean curvature in a sphere, Arch. Math. 61(1993), 489-496.

19. H.-W. Xu: A gap of scalar curvature for higher dimensional hypersurfaces with constant mean curvature, Appl. Math. J. Chinese Univ. Ser. A. 8(1993), 410-419.

20. H.-W. Xu: On closed minimal submanifolds in pinched Riemannian manifolds, Trans. Amer. Math. Soc. 347(1995), 1743-1751.

21. H.-W. Xu and J.-R. Gu: An optimal differentiable sphere theorem for complete manifolds, Math. Res. Lett. 17(2010), 1111-1124.

22. H.-W. Xu and X.-A. Ren: Closed hypersurfurfaces with constant mean curvature in a symmetric manifold, Osaka J. Math. 45(2008), 747-756.

23. H.-W. Xu and L. Tian: A new pinching theorem for closed hypersurfaces with constant mean curvature in $S^{n+1}$, Asian J. Math. 15(2011), 611-630. 
24. H.-W. Xu and E.-T. Zhao: A characterization of Clifford hypersurface, preprint, 2008.

25. H.-W. $\mathrm{Xu}$ and E.-T. Zhao: Topological and differentiable sphere theorems for complete submanifolds, Comm. Anal. Geom. 17(2009), 565-585.

26. Z.-Y. Xu: Rigidity theorems for compact minimal hypersurfaces in a sphere, Bachelor Thesis, S.-T. Yau Mathematics Elite Class, Zhejiang University, 2010.

27. H.-C. Yang and Q.-M. Cheng: An estimates of the pinching constant of minimal hypersurfaces with constant scalar curvature in unit sphere, Manuscripta Math. 84(1994), 89-100.

28. H.-C. Yang and Q.-M. Cheng: Chern's conjecture on minimal hypersurfaces, Math. Z. $\mathbf{2 2 7}(1998)$, 377-390.

29. S.-T. Yau: Submanifolds with constant mean curvature. I, II, Amer. J. Math. 96, 97(1974, 1975), 346-366, 76-100.

30. Q. Zhang: The pinching constant of minimal hypersurfaces in the unit spheres. Proc. Amer. Math. Soc. 138(2010), 1833-1841.

\section{Zhi-Yuan Xu}

Center of Mathematical Sciences

Zhejiang University

Hangzhou 310027, China

E-mail: srxwing@zju.edu.cn 